\title{
Petrofísica Computacional Aplicada a Analise da Tortuosidade de Rochas Carbonáticas
}

Dantas, A.P.T. 1; Vidal, A.D. ${ }^{2}$; Soares, J.A. ${ }^{1}$; Medeiros, L.C. ${ }^{2}$

${ }_{1}$ Laboratório de Petrofísica, Universidade Federal de Campina Grande

2 PETREC - Petroleum Research and Technology

Copyright 2016, SBGf - Sociedade Brasileira de Geofísica

Este texto foi preparado para a apresentação no VII Simpósio Brasileiro de Geofísica, Ouro Preto, 25 a 27 de outubro de 2016. Seu conteúdo foi revisado pelo Comitê Técnico do VII SimBGf mas não necessariamente representa a opinião da SBGf ou de seus associados. É proibida a reprodução total ou parcial deste material para propósitos comerciais sem prévia autorização da SBGf.

\section{Resumo}

A petrofísica computacional se apresenta como um avanço das técnicas de análise de imagens digitais, e se trata de uma metodologia rápida e de baixo custo para a predição de propriedades. As rochas carbonáticas são extremamente heterogêneas e consequentemente mais difíceis de serem caracterizadas, visto que tais rochas abrangem um grande intervalo de tamanho e formas diferenciadas de poros, resultando numa distribuição espacial complexa e com pouca conectividade nos poros. De acordo com Lucia (2007), sedimentos carbonáticos são compostos por partículas que podem mostrar uma ampla variedade de tamanhos, formas e mineralogias. Neste trabalho foi aplicada a técnica de microtomografia computadorizada de raios x com o objetivo de analisar computacionalmente a tortuosidade dos poros de rochas carbonáticas, e sua relação com a porosidade, permeabilidade e superfície específica, as quais também são estimadas a partir da petrofisica computacional.

\section{Introdução}

As características petrofísicas são resultantes de toda a história geológica dos sedimentos que constituem uma rocha, em particular das condições de sedimentação e fenômenos diagenéticos. O trabalho de caracterização petrofísica tem particular interesse nas propriedades que permitem determinar porosidade, permeabilidade e saturação de fluidos em uma rocha reservatório, as quais são utilizadas para identificar, caracterizar e avaliar um reservatório de hidrocarboneto (MELANI, 2015).

A petrofísica computacional se apresenta como um avanço das técnicas de análise de imagens digitais, aplicadas em seções planas de rochas, e se trata de uma metodologia rápida e de baixo custo para a predição de propriedades como porosidade, permeabilidade intrínseca, permeabilidade relativa e parâmetros elétricos como fator de formação e índice de resistividade, a partir unicamente do conhecimento da microestrutura da rocha (FERNANDES et al., 2001). A microtomografia computadorizada de raios- $X$ é uma das técnicas não destrutivas mais adequadas para a investigação da estrutura interna de uma rocha maciça, baseia-se na capacidade dos materiais absorverem a radiação $X$ de forma diferenciada, dependendo da sua densidade e composição química, sendo que sua maior limitação é a baixa resolução atingida, que muitas vezes é da ordem de micrômetros. A integração das seções, ou imagens, obtidas por este método possibilita a visualização de uma estrutura 3D do interior do material amostrado. As rochas carbonáticas são formadas a partir de processos químicos, bioquímicos e aloquímicos envolvendo a deposição e decomposição de organismos ricos em cálcio, como algas, conchas, corais e moluscos (CAMBIER, 2011).

A particularidade e dificuldade de caracterização das rochas carbonáticas também são ressaltadas por Knackstedt et al. (2006), visto que tais rochas abrangem um grande intervalo de tamanho e formas diferenciadas de poros, resultando numa distribuição espacial complexa e com pobre conectividade nos poros. De acordo com Lucia (2007), sedimentos carbonáticos são compostos por partículas que podem mostrar uma ampla variedade de tamanhos, formas e mineralogias. Neste trabaho foram utilizadas imagens de microtomografia de raios $\mathrm{x}$ para a analise computacional da tortuosidade de rochas carbonáticas, e sua relação com a porosidade, permeabilidade e superfície específica. Todas estas propriedades foram estimadas através da técnica de petrofisica computacional.

\section{Seleção e Localização das Amostras}

Neste estudo foram selecionadas um total de 14 (quatorze) amostras de rochas carbonáticas, sendo cinco da Formação Riachuelo (Bacia Sergipe-Alagoas), quatro da Formação Santana (Bacia Araripe) e cinco da Formação Jandaíra (Bacia Potiguar). Os plugs preparados estão descritos na Tabela 1, bem como a denominação aplicada às amostras, de modo a facilitar sua identificação.

Tabela 1. Nomenclatura e denominação dos plugs selecionados no estudo e formação/bacia a que pertencem.

\begin{tabular}{c|c|c}
\hline Amostra & Denominação & Formação/Bacia \\
\hline PC3A_1 & R-1 & Riachuelo/Sergipe-Alagoas \\
\hline PC3C_23_V & R-2 & Riachuelo/Sergipe-Alagoas \\
\hline NAS_1 & R-3 & Riachuelo/Sergipe-Alagoas \\
\hline NAS_8 & R-4 & Riachuelo/Sergipe-Alagoas \\
\hline PC1A_1_1 & R-5 & Riachuelo/Sergipe-Alagoas \\
\hline PT11_H1 & S-1 & Santana/Araripe \\
\hline PT9F_H4 & S-2 & Santana/Araripe \\
\hline PT09_1(6M) & S-3 & Santana/Araripe \\
\hline PT09_1(12M) & S-4 & Santana/Araripe \\
\hline CAL_RONC_V & J-1 & Jandaira/Potiguar \\
\hline AR_OBL_V3 & J-2 & Jandaira/Potiguar \\
\hline HPC_H1 & J-3 & Jandaira/Potiguar \\
\hline ROSARIO_H & J-4 & Jandaira/Potiguar \\
\hline AR_OBL_H1 & J-5 & Jandaira/Potiguar \\
\hline
\end{tabular}




\section{Metodologia}

O processamento das imagens tomográficas foi realizado com o software Avizo Fire $^{\circledR}$ 8.0, para determinar computacionalmente a porosidade, tortuosidade e superfície específica.

A permeabilidade foi estimada utilizando a equação de Kozeny-Carman (TIAB \& DONALDSON, 2004), a partir dos valores de porosidade, tortuosidade e superfície especifica estimados.

\section{Cálculo da Macroporosidade}

Para calcular a macroporosidade, delimita-se uma área da amostra. Em seguida segmenta-se a imagem definindo um limiar de tom de cinza para separar pixels que representam macroporos de pixels que representam a matriz ou grãos. Este limiar é definido de forma específica para cada amostra. Segundo MEDEIROS (2013), é possível obter o volume do espaço poroso (pixels mais escuros que o limiar) e o volume da matriz mineral (pixels mais claros que 0 limiar). A macroporosidade $(\mathrm{m} \phi)$ do subvolume pode ser medida de acordo com a equação (1):

$$
\begin{gathered}
m \Phi=\frac{n e}{n t} \\
n t=n e+n i
\end{gathered}
$$

Onde ne é o número de pixels de material exterior ou espaço poroso, ni é o número de pixels de material interior ou matriz mineral e $n t$ corresponde ao número total de pixels.

\section{Cálculo da microporosidade}

A microporosidade são os poros contidos na amostra que estao abaixo da resolução obtidas nas imagens e desta forma nao são claramente identificados. Para amostras carbonaticas é comum este tipo de porosidade, oriunda de processos secundários. Para o calculo da microporosidade,. delimita-se uma área da amostra e a converte para64 bits, formato necessário para a realização do cálculo aritmético ao qual será submetida posteriormente. Em seguida segmenta-se a imagem, para definir a fase intermediária, a qual contém a microporosidade. Com os limiares definidos, aplica-se a ferramenta de cálculo aritmético e a equação (3) é utilizada para o cálculo da microporosidade $(\mu \phi)$, AL RATROUT et al. (2013).

$$
\mu \Phi=b \times\left(1-\frac{(a-L p)}{(L s-L p)}\right)
$$

Em $a$ é adicionada a imagem original convertida, em $b$ a imagem correspondente da fase intermediária também convertida, $L p$ e $L s$ repressentam respectivamente 0 limiar dos poros e o limiar do sólido. Obtem-se como resultado do cálculo aritmético, valores que permitem estimar a microporosidade através da média da microporosidade nas imagens.

Assim a microporosidade final $(\mu \phi f)$ pode ser calculada de acordo com a equação (4):

$$
\mu \Phi f=\frac{V f i}{V t} \times m
$$

Onde Vfi corresponde ao volume da fase intermediária ou material interior, $m$ é a média da microporosidade nas imagens e $V t$ é o volume total da imagem, calculado através de suas dimensões e resolução.

\section{Cálculo de tortuosidade e superfície específica}

A tortuosidade é estimada a partir de um módulo que calcula o quão tortuoso é um caminho, de uma imagem binarizada 3D, ao longo do eixo $z$.

Primeiro é calculado o centróide de cada plano da imagem, em seguida, calcula-se o comprimento do caminho através destes centróides e depois divide-se pelo número de planos ao longo do eixo z. A tortuosidade é então, a razão entre o comprimento de uma curva e a distância entre as suas extremidades.

O cálculo da superfície específica, por sua vez, é realizado através de uma ferramenta que estima a relação entre a superfície binarizada e o volume total do modelo . No caso de um modelo 3D, é calculada como a divisão da área 3D pelo volume 3D.

\section{Cálculo da permeabilidade}

A permeabilidade computacional das amostras foi calculada, através de equação de Kozeny-Carman (TIAB \& DONALDSON, 2004), de acordo com a equação (4).

$$
\mathrm{k}=\left(\frac{1}{2 \tau \mathrm{S}_{\mathrm{vgr}}^{2}}\right) \frac{\phi^{3}}{(1-\phi)^{2}}
$$

Onde: $k=$ permeabilidade $\left(\mu \mathrm{m}^{2}\right)$;

$\Phi=$ porosidade computacional(-);

$\mathrm{T}=$ tortuosidade computacional(-);

Svgr computacional = superfície específica do material poroso, ou a área total exposta no espaço poroso por unidade de volume de grão $(1 / \mu \mathrm{m})$. 


\section{Resultados}

Para validação das simulações computacionais foram plotados valores de porosidade e permeabilidade estimados computacionalmente e os valores obtidos em laboratório, conforme mostrado na Figura 1 e 2. Observou-se que os valores estimados tendem a ser menores que os valores obtidos em laboratório, indicando que nem todo o espaço poroso dos plugues foi representado pelas subamostras imageadas, devido às heterogeneidades desse tipo de material.

Além disto, a resolução das imagens obtidas pela microtomografia é, na maioria das vezes, insuficiente para alguns tipos de rochas. Nas amostras estudadas, as resoluções variaram entre 1,24 e 2,56 $\mu \mathrm{m}$, podendo não ter sido suficiente para poros que se encontram abaixo deste intervalo de resolução.

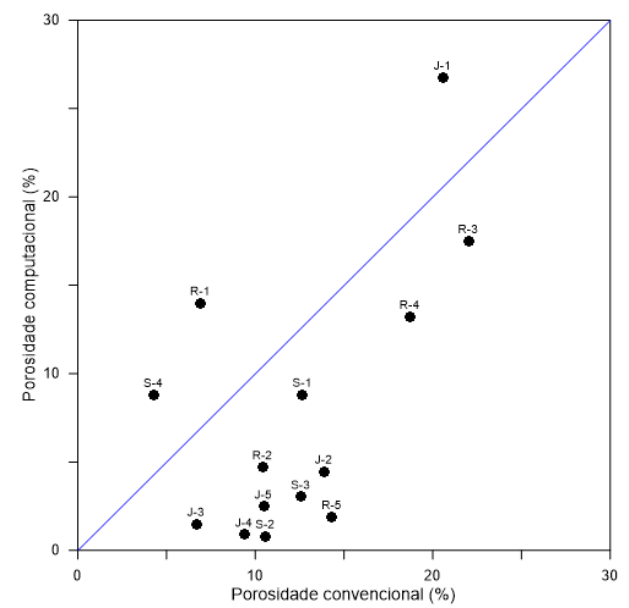

Figura 1: Relação entre porosidade convencional e porosidade computacional para as amostras estudadas.

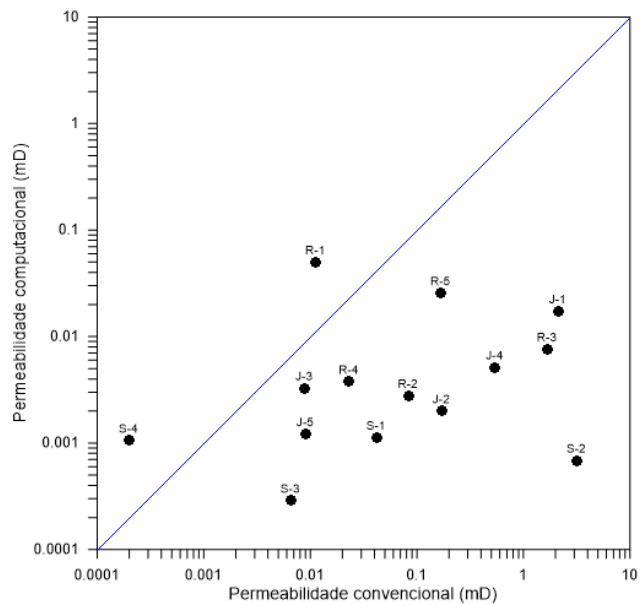

Figura 2: Relação entre a permeabilidade convencional e permeabilidade computacional para as amostras estudadas.
Para maiores valores de macroporosidade e microporosidade, os resultados obtidos para a tortuosidade tendem a ser menores conforme mostrado nas Figuras 3 e 4 . Isto se repete também para valores de porosidade total (Figura 5), mostrando que quanto mais porosa for à rocha, menor sera a complexidade do meio poroso, facilitando assim, o escoamento de fluidos.

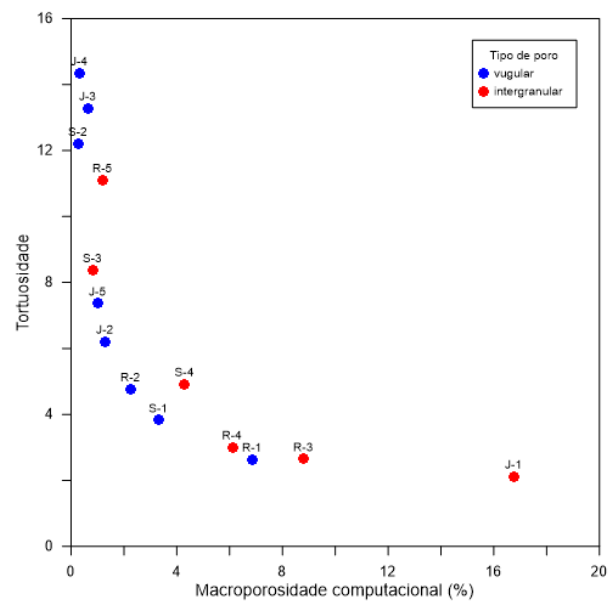

Figura 3: Relação entre macroporosidade e tortuosidade para os valores obtidos computacionalmente.

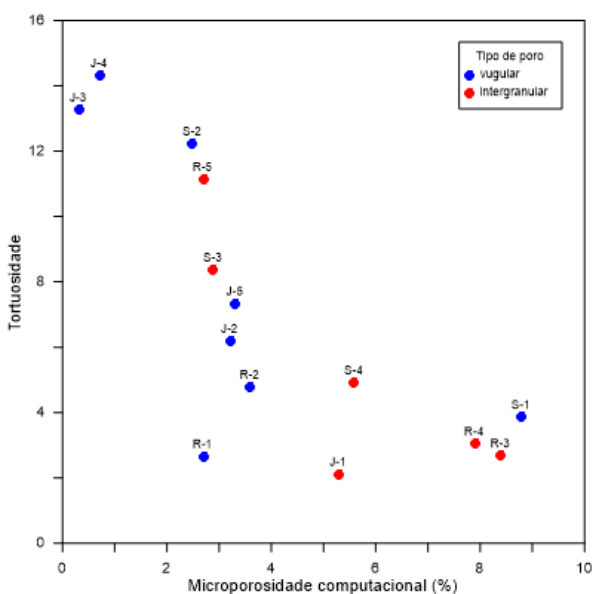

Figura 4: Relação entre microporosidade e tortuosidade, para os valores obtidos computacionalmente. 


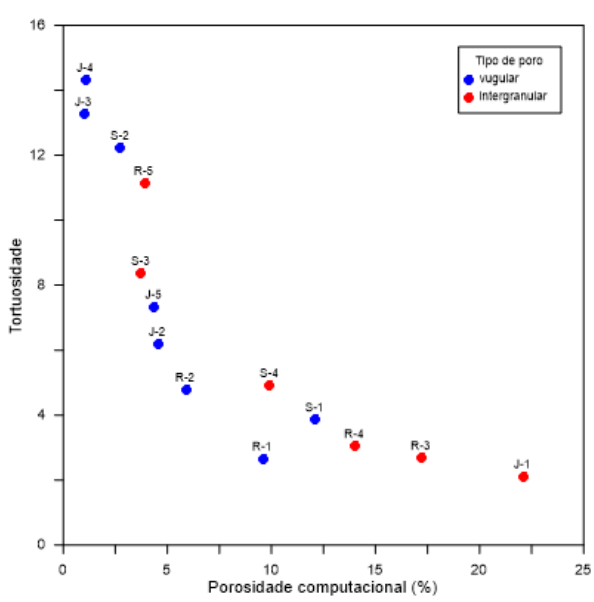

Figura 5: Relação entre porosidade total e tortuosidade, para os valores obtidos computacionalmente.

A Figura 6 apresenta os resultados obtidos computacionalmente para superfície específica dos grãos (Sogr) e tortuosidade. Observa-se uma tendência de aumento dos valores de tortuosidade à medida que Sogr diminui. Esta tendência se mostra de acordo com o tipo de poro encontrado nas rochas. Para porosidade vugular essa tendência ocorre mais bruscamente, enquanto que para porosidade intergranular a variação é mais gradual e lenta.

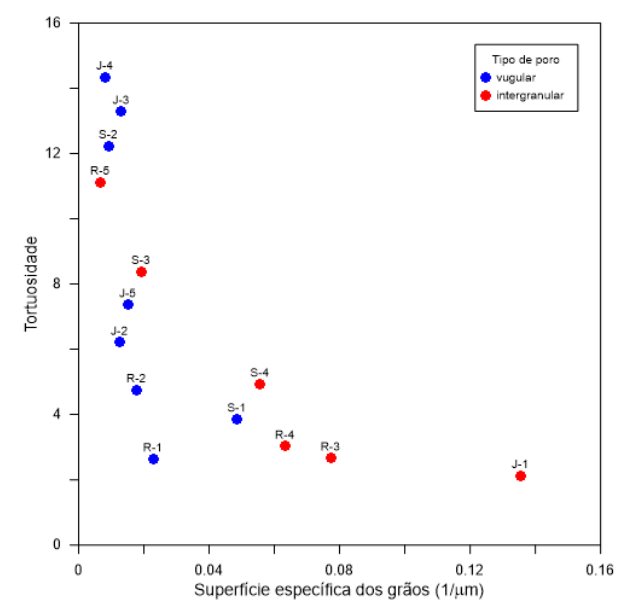

Figura 6: Relação entre a superfície específca dos grãos e tortuosidade.

Na Figura 7 é mostrado o gráfico de permeabilidade versus superfície específica dos poros. Os resultados obtidos mostram uma tendência de aumento da permeabilidade quando a superfície específica dos poros diminui.

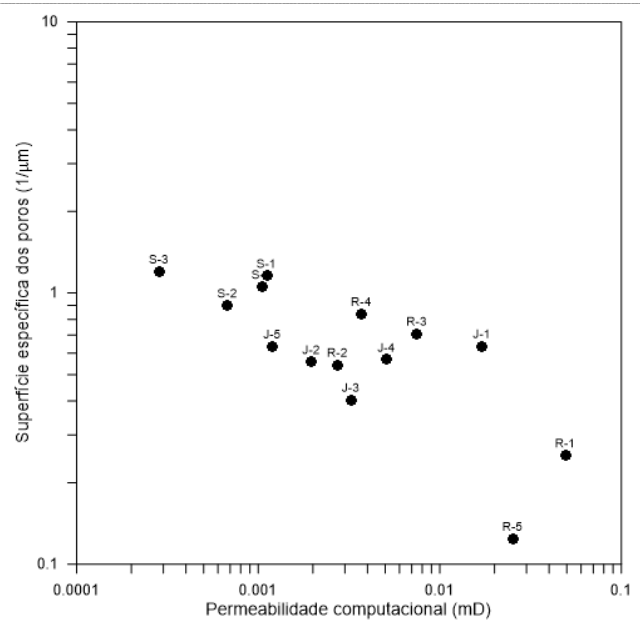

Figura 7: Relação entre a permeabilidade computacional e superfície específica dos poros.

\section{Discussão e Conclusões}

Para a analise computacional da tortuosidade em relação a outras propriedades físicas da rocha (porosidade, permeabilidade e superfície especifica) foram inicialmente comparados os dados de porosidade e permeabilidade obtidos computacionalmente e em laboratório. As diferenças entre os resultados ocorreu devido a não representatividade das subamostras extraidas. Uma opção seria a extração de uma quantidade maior de subamostras para caracterizar de forma mais precisa as heterogeneidades do plugue.

De modo geral pode-se concluir que a tortuosidade das rochas carbonáticas depende de muitas variáveis e propriedades, como descrito abaixo:

- $\quad$ Porosidade: quanto maior o valor da porosidade total da amostra, menor a sua tortuosidade. Esta relação varia se for levado em consideração o tipo de porosidade e tamanho de poros. Uma rocha com porosidade do tipo intergranular tende a apresentar valores menores de tortuosidade se comparado com uma amostra que possui porosidade do tipo vugular.

- Permeabilidade: rochas de maior permeabilidade tendem a apresentar valores menores de tortuosidade. No entanto, rochas com baixa permeabilidade tanto podem apresentar valores baixos quanto altos de tortuosidade, dependendo do tipo de porosidade, já que esta influencia diretamente na conectividade da amostra.

- Superfície Específica: há um aumento da tortuosidade quando a superfície específica dos poros diminui. 


\section{Referências}

ASSINE, M. L. Análise Estratigráfica da Bacia do Araripe, Nordeste do Brasil. Revista Brasileira de Geociências, São Paulo, v.22, n. 3, p. 289-300, 1992.

BERRYMAN, J.G.; WANG, H.F. Elastic wave propagation and attenuation in a double-porosity dual-permeability medium. International Journal of Rock Mechanics and Mining Sciences, v. 37, p. 63-78, 2000.

BLAUTH, M.; FARIA, R. T.; MAUL, A. R.; MONTEIRO, M. C.; FRANCO, M. P.; CARNEIRO, S. R. R.; OLIVEIRA, R. M.; TIBANA, P. Workflow for Geological Characterization and Modeling of the Albian Carbonate Reservoirs from Offshore Campos Basin, Brazil. New Understanding of the Petroleum Systems of Continental Margins of the World. 32nd Annual GCSSEPM Foundation Bob F. Perkins Research Conference, p. 365-380. Houston - TX, 2012.

CAMBIER, P. H. M. M. G. Simulação Computacional Tridimensional da Formação e Evolução de Plataformas Carbonáticas. Dissertação de Mestrado. Pontíficia Universidade Católica do Rio de Janeiro, Rio de Janeiro, 2011.

CASSAB, R. C. T. Paleontologia da Formação Jandaíra, Cretáceo Superior da Bacia Potiguar, com ênfase na paleobiologia dos gastrópodos. Programa de PósGraduação em Geologia, Instituto de Geociências, 184 p. Universidade Federal do Rio de Janeiro, Rio de Janeiro, 2003.

COSENTINO, L. Integrated Reservoir Studies. Editions Technip, 336p, Paris, 2001.

DANTAS, C. X. G. Estudo Geodinâmico e Evolução Tectono-Estrutural de Parte das Bacias de SergipeAlagoas (Região entre Igreja Nova-AL e Propriá -SE) no Mesozóico. Dissertação de Mestrado. Pós-Graduação em Geociências. Universidade Federal de Pernambuco, 2011.

DULIU, O. Computer axial tomography in geosciences: an overview. Earth Science Reviews, v. 48, p. 265-281, 1999.

FERNANDES, C. P.; SANTOS, L. O. E.; PHILIPPI, P. C.; BUENO, A. D.; RODRIGUES, C. R. O.; GASPARI, H. C. Predição de propriedades petrofísicas de rochas reservatório de petróleo a partir de análise de imagens. 1ํ Congresso Brasileiro de P\&D em Petróleo e Gás, UFRN - SBQ Regional RN, Natal, 2001.

HASUI, Y.; CARNEIRO, C. D. R.; ALMEIDA, F. F. M.; BARTORELLI, A. Geologia do Brasil. Editora Beca, 900 p. São Paulo, 2012. 